\title{
Traffic Performance of U-Turn Effects at Median Opening on Four-Lane Divided of Urban Street (Study Case: Yogyakarta, Indonesia)
}

\author{
Prima J. Romadhona ${ }^{1, *}$, Bachnas ${ }^{1}$, Lantika Nurkumala ${ }^{1}$ \\ ${ }^{1}$ Department of Civil Engineering, Universitas Islam Indonesia, Indonesia
}

\begin{abstract}
The main purpose of this study was to determine traffic performance at a median opening on the urban street. In order to achieve the research purpose, data were obtained at four U-turn locations in sequence at Gejayan area of Yogyakarta. By using VISSIM software, it analyzed traffic delay, queue length, and average travel speed on the existing condition then suggested the solution to improve the performance. This research showed that the longest queue length was around $200 \mathrm{~m}$ and the delay was almost $25 \mathrm{~s}$. The result suggested that U-turn location with bad performance should be omitted. Reducing the number of U-turn locations will improve more than $50 \%$ of the traffic performance in the urban street. In addition, $100 \mathrm{~m}$ should be the minimum distance among $\mathrm{U}$ turns to maintain the traffic performance.
\end{abstract}

\section{Introduction}

Median is a section of road which is located in the middle of the road and not used for vehicular traffic. The main function of the median is to separate traffic flow in the opposite direction and reduce conflict area for vehicles. In addition, the median also acts as a speed change and deceleration/acceleration lane for right turn traffic and U-turn. Basically, vehicles making a U-turn at a median opening are decelerating their speed thus will affect the traffic flow in the same direction. However, since the limited maneuver area makes short radius of the road geometry, it causes vehicles to be unable to make a direct U-turn. This condition leads other vehicles to be impaired or even stopped either from the same direction or the opposite.

For instance, Liu et al. [1] estimated the potential capacity of U-turns at unsignalized median openings on six-lane streets. The results of Liu, et al in [1] suggest that the Highway Capacity Manual can be applied to estimate U-turn capacity in the research area. They also built a procedure to estimate the capacity of U-turn movement at median openings on multilane highways. The research result by Liu et al at [2] found that vehicles making U-turns at median openings with wide medians (median nose width $\geqslant 6.4 \mathrm{~m}$ ) have the larger capacity than those making U-turns at median openings with narrow medians

*Corresponding author: prima_dhona@uii.ac.id 
(median nose width $<6.4 \mathrm{~m}$ ). Zhou et al. said in [3] that the location of U-turn median openings has a great impact on the operations of U-turns.

Mohapatra et al at [4] identified the conflict zone between a turning vehicle and oncoming vehicles at uncontrolled median openings on urban roads. A simple model was proposed to identify the boundary of the conflict zone at median openings. A geometrical augmentation required at the median opening was suggested. Mohapatra et al in [5] also said that the conflicting traffic on six-lane roads was found to be relatively low, but on fourlane roads, almost all of the opposing traffic becomes conflicting traffic for U-turns.

However, as stated by Liu et al in [6] that every movement at U-turn was very interesting to visualize by traffic simulation program. VISSIM, as one of the traffic simulation programs, provides reasonable capacity estimation for U-turn at unsignalized intersection with raised median cross sections, after crucial parameters were properly defined and calibrated. According to Yang and Zhou [7], a U-turn movement simulation using other software program indicates that the delay, as well as the travel time of direct travel time, was higher than without using U-turn. Hummer and Reid [8] determined the importance of arterial geometry related to total system time, average stops per vehicle, and average speed. The result showed that the median U-turn and super street median can improve the travel time and average speed compared with the traditional two-way left turn lane. Della et al. [9] made a simulated case study using VISSIM with different width median openings and the result showed that, in narrow width median opening, it will lead to major delay and long travel time. Pirdavani et al made an innovation to improve the road performance by fly-over U-Turn then made the traffic simulation by VISSIM. They defined that median opening U-turn has number of delay than fly-over U-turn. Other innovation of U-turn should be built for example new types of U-turn intersection which are geometrically designed with a raised island providing a protected U-turn movement. Pirdavani et al [10] found that this new type of U-turn facility commonly produces shorter travel time.

In this study, there was a two-way four-lane street with a median that has more than 5 U-turn locations in the 3-km road length. Gejayan area of Yogyakarta City is one of the leading business districts that have a high traffic flow. There are many points that accommodate median opening and U-turn or right turn. Some vehicles cannot pass the Uturn perfectly so as to disturb the traffic in the same direction and cause queues. Since the distance between U-turn locations at median opening is very close, there are long queues that often occur along the street.

Therefore, it is necessary to find the influence of U-turn movement by determining the traffic delay at median opening on the urban street and appropriate problem-solving for optimizing the traffic performance. In this research, there were 4 U-turn locations in sequence along the urban street at Yogyakarta City. The locations were divided by some segments as research locations can be seen in Figure 1. The detailed geometry can be seen in Figure 2 as follows. 


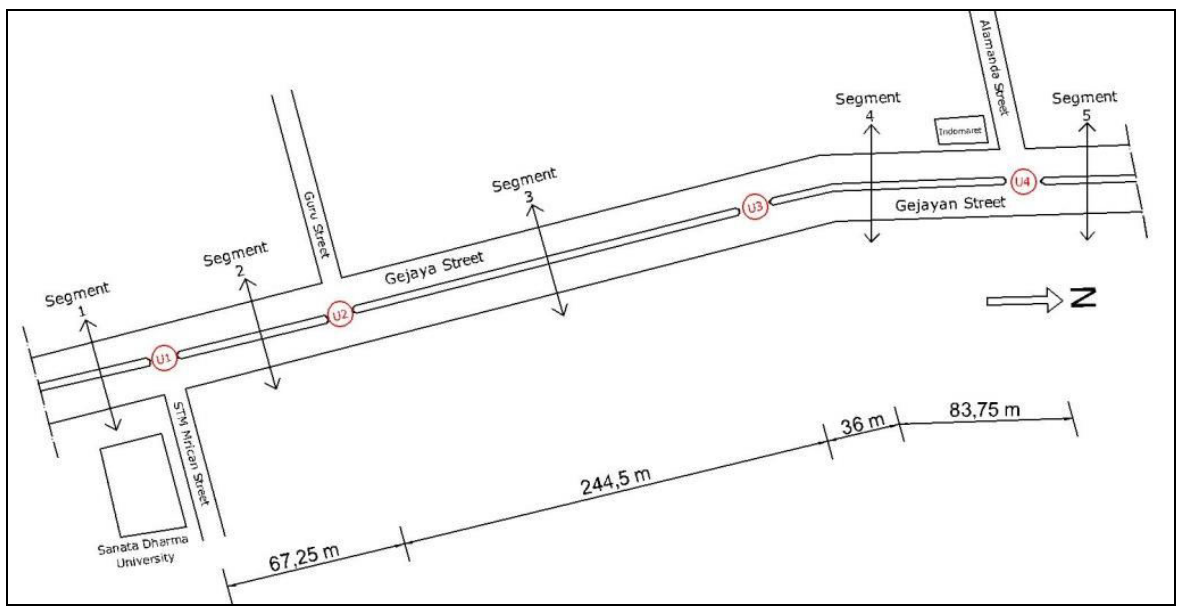

Fig 1.U-turn Locations in Sequence along the Urban Street

\section{Research Methodology}

\subsection{Data Collection}

The four-lane two-way urban street with median at the research location was divided into some segments with 4 U-turn locations in sequence. Primary data was taken for this research in collecting the road geometry, traffic volume, and volume of U-turn traffic. The position of the surveyor can be conceived in Figure 3. The surveyor position was illustrated at A-D. The distance of A and B was 50 meters. Two surveyors were placed at $\mathrm{A}$ and $\mathrm{B}$ to count the number and time of vehicles that did U-turn. The data collected in the opposite direction were calculated at $\mathrm{C}$ and $\mathrm{D}$. The surveyor position applied to all the research locations.

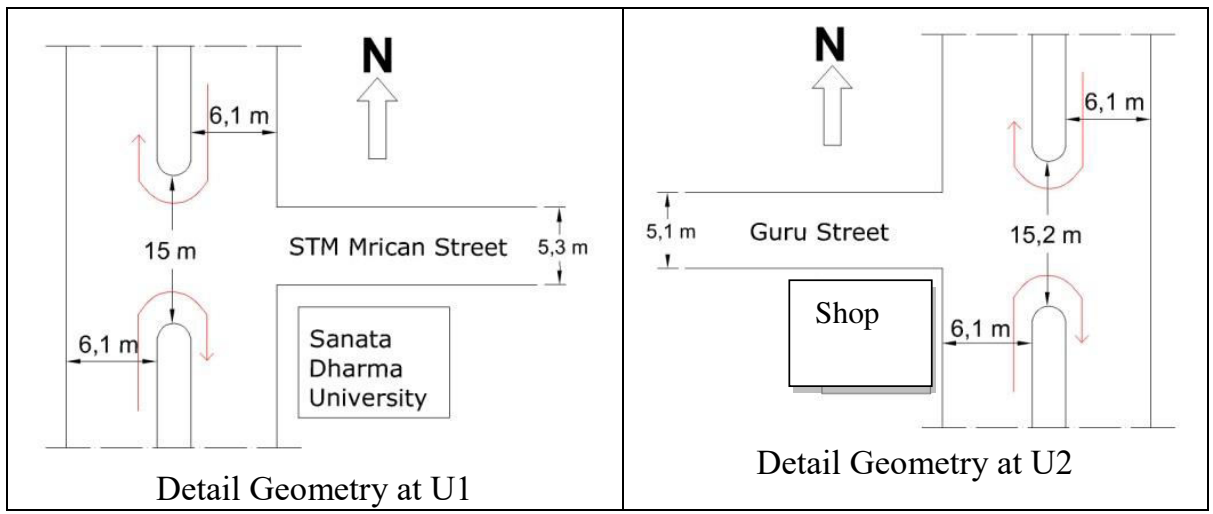




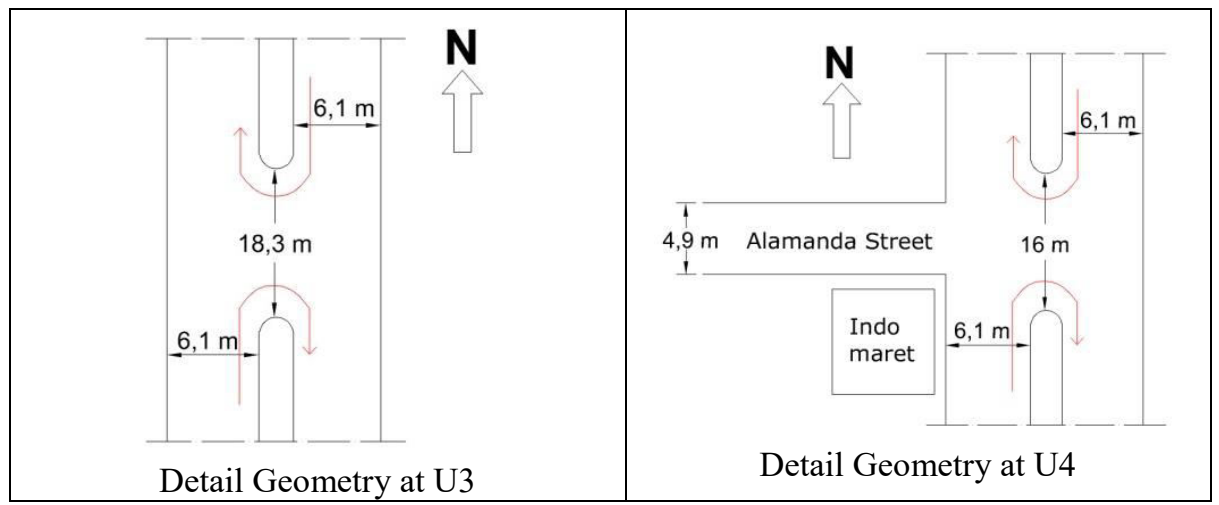

Fig 2. Detailed Geometry at U1-U4

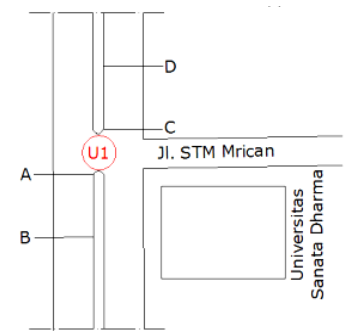

Fig 3. Surveyor position at U-Turn 1

\subsection{Traffic Simulation}

Traffic evaluation was analyzed to calculate the length of queues and delays on road as a result of the U-turn motion at the median opening. The traffic simulation was conducted using VISSIM software. After getting the results of the existing condition, some scenarios to improve the traffic performance were simulated. Still, the traffic simulation in solving the problems used VISSIM software as a way of the engineer to find the most ideal conditions.

\section{Traffic Performance on Existing Condition}

\subsection{Traffic Volume and Characteristic}

The highest peak hour volume was obtained on Monday Afternoon at 16:30 to 17:30 with 5,559 vehicles/hour in the North-South direction and 3,072 vehicles/hour in the SouthNorth direction. Motorcycles (MC) showed the highest user in all segments. Meanwhile, the highest volume of traffic occurred in the U4 from North to South direction. In general, a number of heavy vehicles and unmotorized were the lowest popular user in this area. The detail volume can be seen in Figure 4 below. 


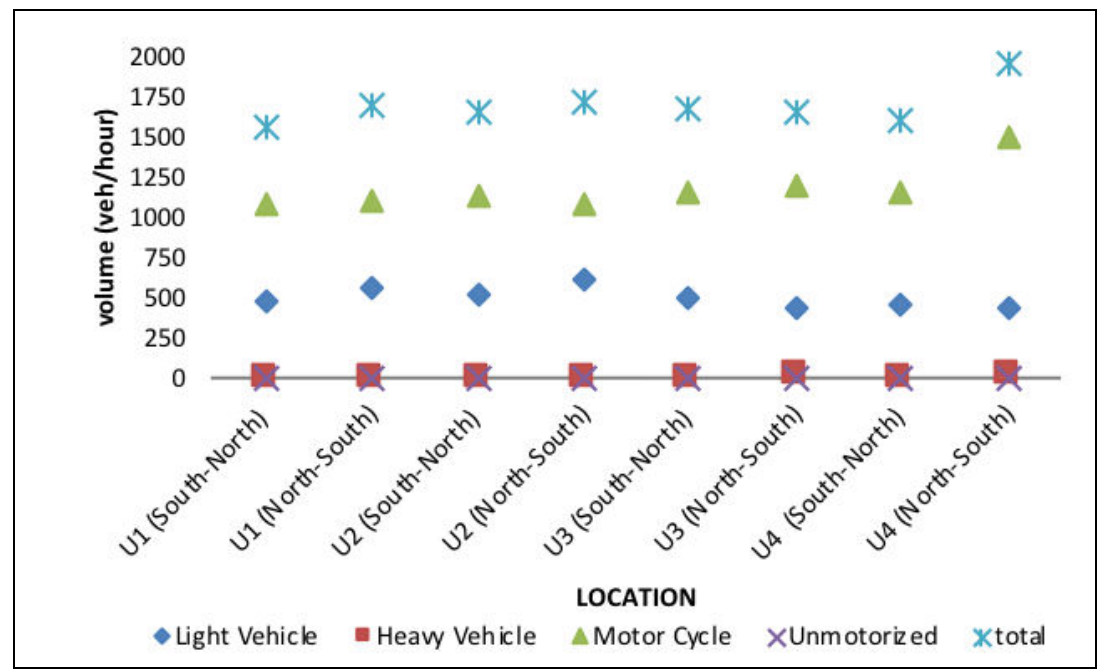

Fig 4. Number of Vehicle Volume at Each Segment

At the peak hour, the number of vehicle doing U-turn at the research locations can be seen in Figure 5 as follows. The busiest location of vehicles doing U-Turn was at U4 where there were shopping areas around the location. Mostly, the number of vehicles doing UTurn at the peak hour was less than 100 vehicles/hour. Besides, as can be seen in Figure 6, motorcycle (MC) was the vehicle with the most U-turns as well as the most frequently used vehicle in Indonesia.

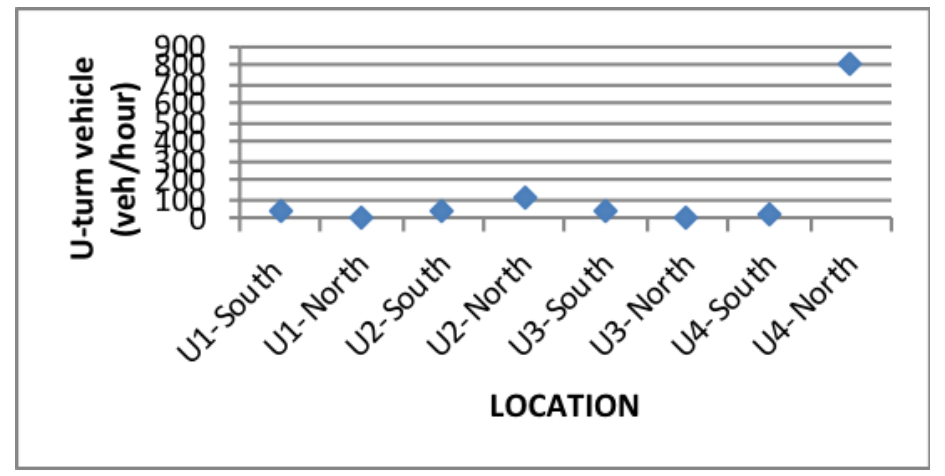

Fig 5. Number of Vehicle Doing U-Turn
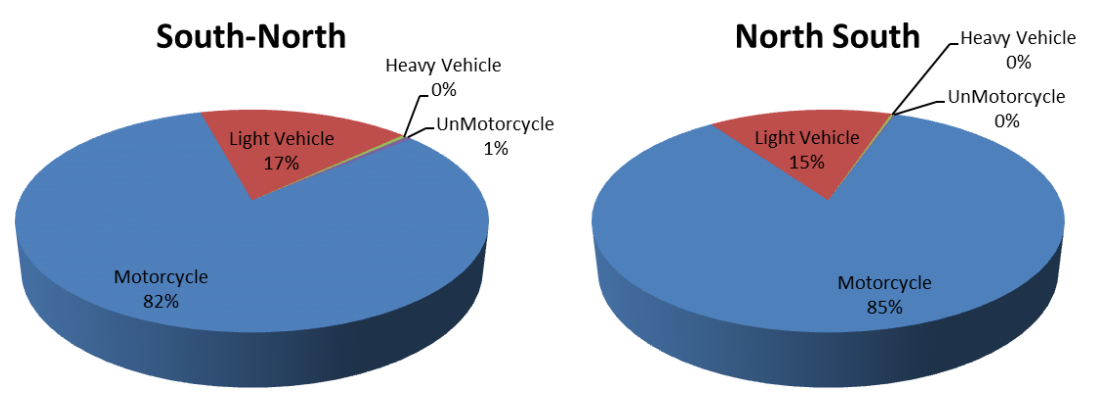

Fig 6. The Composition of Vehicles Doing U-Turn 
In order to make accurate traffic simulation from VISSIM, a parameter calibration was made. Since the driving behavior in a developing country such as Indonesia is different, some calibrations were made such as the desired position at free flow, distance standing and driving, etc. As a result, the difference of the traffic volume between the data and VISSIM simulation was less than $6.5 \%$. The detailed result can be seen in Table 1 as follows.

Table 1. Traffic Volume Validation of VISSIM

\begin{tabular}{|l|c|c|c|}
\hline \multicolumn{1}{|c|}{ Location } & $\begin{array}{c}\text { VISSIM } \\
\text { (vehicle/hour) }\end{array}$ & $\begin{array}{c}\text { Field Data } \\
\text { (vehicle /hour) }\end{array}$ & Difference (\%) \\
\hline Gejayan Street (S-N) & 3057 & 3072 & 0.49 \\
\hline Gejayan Street (N-S) & 5422 & 5559 & 2.46 \\
\hline STM Mrican Street & 811 & 808 & 0.37 \\
\hline Gang Guru & 460 & 443 & 3.84 \\
\hline Gang Alamanda & 479 & 451 & 6.21 \\
\hline
\end{tabular}

Since the traffic volume result indicates the field data, the model was used as the traffic simulation to find the traffic performance in the existing condition and future proposed scenario.

\subsection{Queue Length and Delay}

As stated by Dastidar and Adeli at [11] that it was important to track the queue length to analyze the congestion characteristics. Still, the delay and queue length were important to measure the traffic performance. In addition for the traffic simulation, as the past research by Liu et al [12], it was possible to count the queue length by an equation model. Traffic simulation by software to find the queue length was counted. By the previous research of Mystkowski and Adeli [13], some programs produced a result 70 to 85 percent of the time, while some others produced a result 50 to 85 percent of the time. Instead of traffic simulation, Murat et al [14] said that regression modeling can be used to find the delay at isolated signalized intersection.

Table 2. Queue Length and Delay of Existing Condition Using VISSIM

\begin{tabular}{lccc}
\hline \multicolumn{1}{c}{ Queue Counter } & Q Length $(\mathbf{m})$ & Q Length Max (m) & Delay (sec) \\
\hline 1: U1-South & 133.787 & 384.306 & 23.372 \\
\hline 2: U1-North & 9.764 & 55.796 & 3.105 \\
\hline 3: U2- South & 0.086 & 26.627 & 1.622 \\
\hline 4: U2- North & 205.940 & 470.202 & 13.144 \\
\hline 5: U3- South & 0.096 & 23.817 & 0.776 \\
\hline 6: U3- North & 30.406 & 138.868 & 8.371 \\
\hline 7: U4- South & 1.153 & 72.002 & 2.499 \\
\hline 8: U4- North & 82.975 & 318.914 & 7.075 \\
\hline
\end{tabular}

As presented in Table 2 concerning traffic simulations at the U-Turn in sequence, the longest delay and queue length occurred at the U-Turn with alley access to the major street. The shortest delay and queue only occurred at U3 location without any access nuisance from the alley. 


\section{Discussion}

\subsection{Traffic Performance Analysis on Existing Condition}

Based on the Guidelines of the Minister of Transportation of Indonesia [15] concerning the management and activities of traffic engineering, the traffic performance was determined by the speed parameter. From the result of the traffic simulation by VISSIM, speed was used as an average travel speed. Value of travel time was taken from vehicle travel time result with the observation distance of 50 meters on each installation of vehicle travel time counter. The detailed result of travel speed can be seen in Figure 7. From the figure, it is important to note that U3 has the highest speed location. However, on the average, U1 has the worst performance since the average speed in both directions were $17 \mathrm{~km} /$ hour. Overall, the average travel speed from the south direction at the research location was better than from the north.

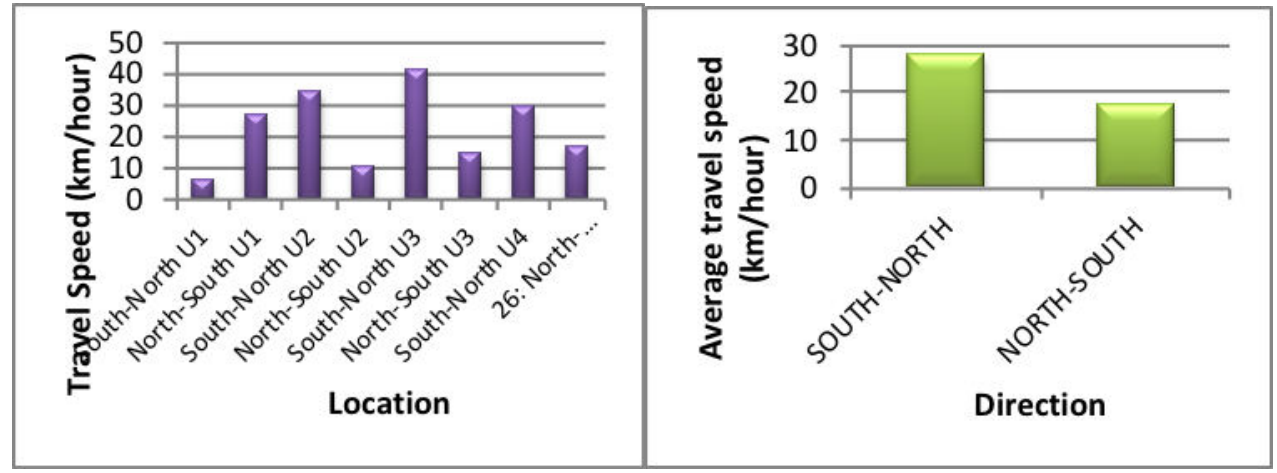

Fig 7. Travel Speed on Existing Condition

\subsection{Alternative Solution I}

In order to increase the traffic performance, some solutions were proposed. Alternative I was moved the median opening 2 (U2) as far as 60 meters to the north. This solution was selected because the access from the alley -at the west side of the main road- at U2 makes the traffic worse. In addition, the distance between the median opening 1 (U1) and median opening 2 (U2) was relatively close to each other (67.2 meters). This solution will allow the traffic from the alley to switch to the south at the extension location of U-turn 2 (U2). The detailed geometry before and after of the solution can be seen in Figure 8. 

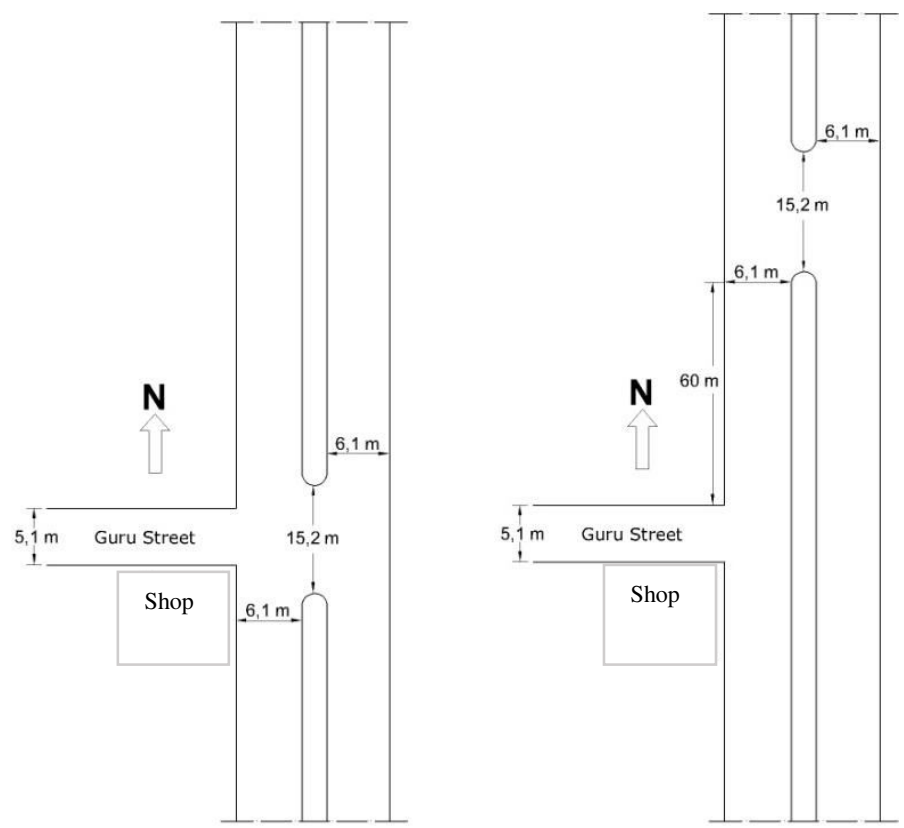

Fig 8. Before (left) and After (right) of Alternative 1

\subsection{Alternative Solution II}

Alternative II was designed by closing two U-Turn in the middle of the sequence of UTurn. As an alternative, median opening 2 (U2) and median opening 3 (U3) were closed so that the traffic flow was diverted to median opening 1 (U1) and median opening 4 (U4). Besides, the traffic flow was lower than the others. The detailed geometry on alternative II can be seen in Figure 9 and 10 .

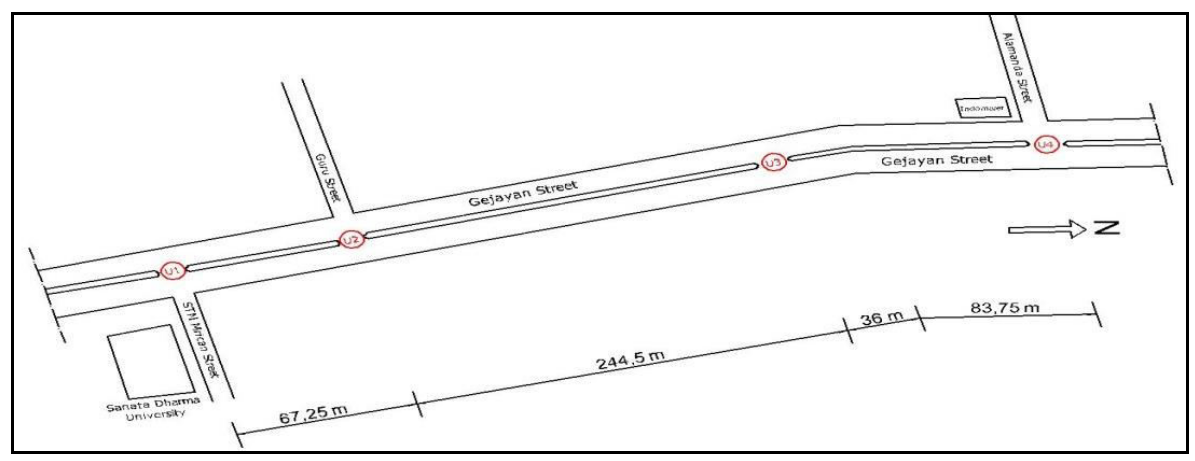

Fig 9. Geometry of Alternative 2 (Before) 


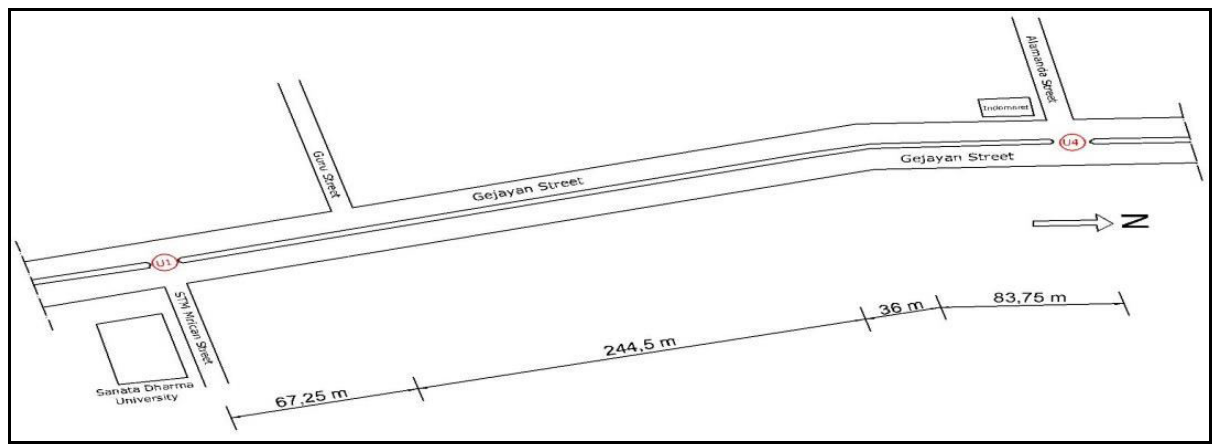

Fig 10. Geometry Alternative 2 (After)

\subsection{Results of Alternative Solutions}

From the proposed solution, the traffic performance was analyzed to find the alteration of traffic. As shown in Figure 11, the queue length and delay in the alternative solutions mostly decrease to $40 \%$ approximately. The queue length of alternative I was reduced significantly at U1 and disappeared at U2 and U3 indeed. Besides, the delay of the solutions decreases by more than $50 \%$. Yet, the queue at U4-A was getting worse slightly in all the proposed solutions. In addition, the delay was increased less than $36 \%$ at U3 on alternative I and at U1 on alternative II.

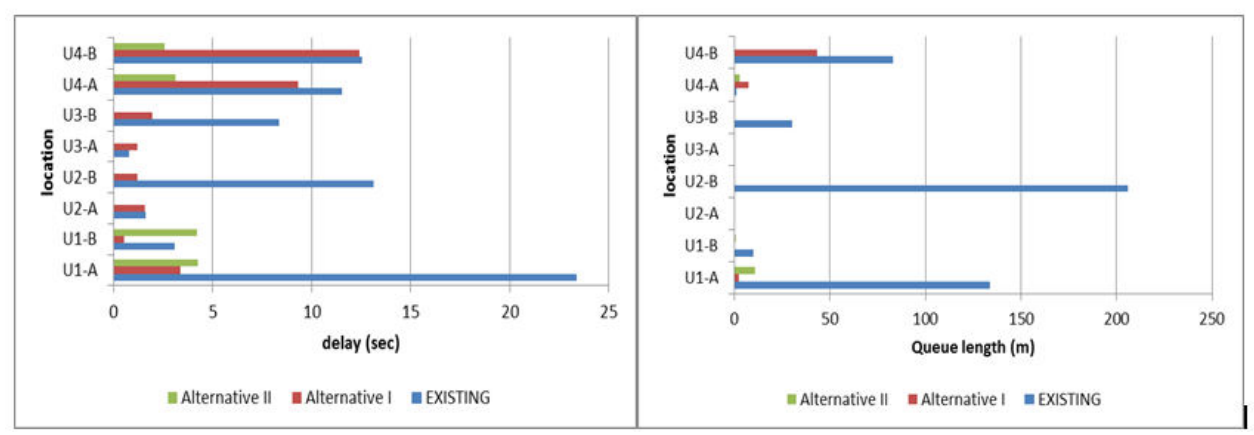

Fig 11. Delay and Queue Length of Alternative Solutions

However, the travel speed analysis for all the alternatives can be seen in Figure 12. From the figure, it can be concluded that the speed increase significantly as much as $40 \%$ on alternative I and $64 \%$ on alternative II. On the average, both alternatives can improve the speed by more than $50 \%$. 


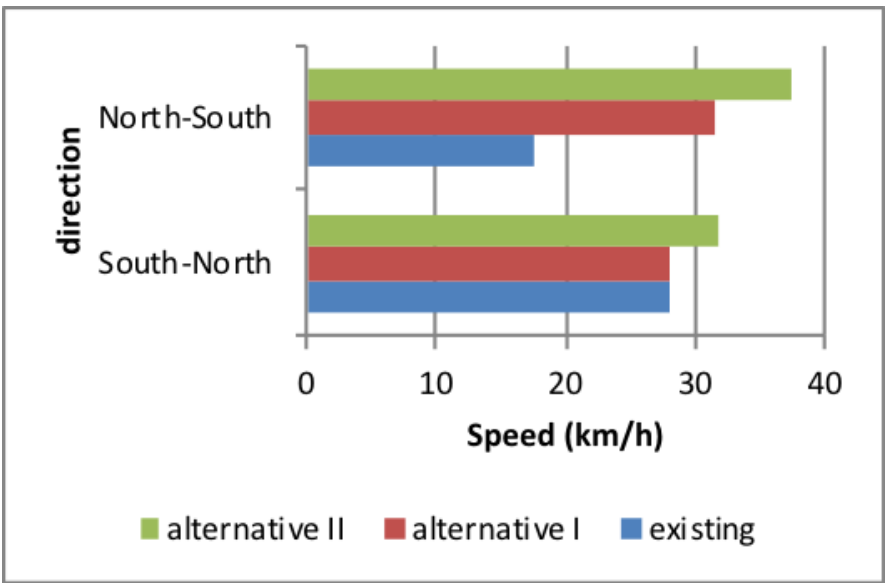

Fig 12. Delay and Queue Length of Alternative Solutions

\section{Conclusion}

U-Turn locations at the median opening along a street with less than $100 \mathrm{~m}$ distance in sequence will lead to bad performance of traffic. This research shows that the longest queue length was around $200 \mathrm{~m}$ and the delay was almost $25 \mathrm{~s}$.

The result suggests that the U-turn location with bad performance should be omitted so as to reduce the number of the U-turn locations facing the aisle will improve more than $50 \%$ traffic performance at the urban street. In addition, $100 \mathrm{~m}$ should be the minimum distance between U-turn locations at the median opening to maintain the traffic performance.

\section{References}

1. P. Liu, J. Lu, and B. Cao. J. T. R. B. 2130 (2008)

2. P. Liu, J. Lu, F. Hu, and G.J. Sokolow, J. T. E. 134, 4, 147-154 (2008)

3. H. Zhou, P. Hsu, J. Lu, and J. Wright. J. T.R. B, 1847, 36-41 (2003)

4. S.S. Mohapatra, P.D. Partha, S. Chandra. J. C. E. 20, 1, 411-420 (2016)

5. S.S. Mohapatra, P. Dev, and S. Chandra. Proceedings of The Institution of Civil Engineers - Transport, 10.1680/JTRAN.14.00089,195-204 (2016)

6. P. Liu, X. Qu, H. Yu, W. Wang and B. Cao. J. T. E. 138(11): 1333-1339 (2012)

7. X. K. Yang, and G. H. Zhou, J. T. E, 130, No. 1, 68-75 (2004)

8. J. Reid, and J. Hummer, J. T. R. B, 1678, 208-215 (1999)

9. R. Della, A. J. Hanafiah, R. Artiansyah. Proceedia Engineering, 125, 461-466 (2015)

10. A. Pirdavani, T. Brijs, T. Bellemans, G. Wets, and V. Koen. Proceeding of International Conference on Urban, Regional Planning and Transportation, 1-6 (2009)

11. G.S. Dastidar, and H. Adeli. J. T. E. 132, 4 (2006)

12. H. X. Liu, X. Wu, W. Ma, H. Hu. J. T. R. 17, 4, 412-427 (2009)

13. C. Mystkowski, and S. Khan. J. T. R. B. 1683, pp 110-117 (1999)

14. Y. S Murat, S, Kutluhan. and Z. Cakici, Proceedia-Social and Behavioral Sciences, 111, 252-261 (2014)

15. Minister of Transportation, The Guidelines of the Management and Activities of Traffic Engineering, Republic of Indonesia, PM 96 (2015) 